\title{
A NOVEL FAULT DIAGNOSIS METHOD FOR ROTATING MACHINERY BASED ON EEMD AND MCKD
}

\author{
Lv, Z.-L.; Tang, B.-P. ; Zhou, Y.\& Zhou, C.-D. \\ State Key Laboratory of Mechanical Transmission, Chongqing University, Chongqing 400030, China \\ College of Mechanical and Power Engineering, Chongqing University of Science and Technology, \\ Chongqing 401331, China \\ E-Mail: bptang@cqu.edu.cn (" Corresponding author)
}

\begin{abstract}
Incipient fault diagnosis of rotating machinery has received extensive research attention for years. However, the diagnosis remains a difficult problem since the incipient faults are generally quite weak in noisy environments. In the present work, a novel fault diagnosis method based on ensemble empirical mode decomposition (EEMD) and maximum correlated kurtosis deconvolution (MCKD) is proposed in order to solve this problem. For the incipient fault of rotating machines, EEMD is firstly performed and the signal is reconstructed based on the calculation correlation coefficient and kurtosis. The impulsive components of faults can be enhanced using the MCKD-based adaptive method, and the weak fault features can be extracted from the envelope spectrum. Finally, the diagnosis results are output. The experimental results indicate that, using the proposed method, the fault impulsive components in the obtained intrinsic mode functions (IMFs) with EEMD can be adaptively enhanced, and the weak fault signal hidden in the noise can be effectively detected.

(Received, processed and reviewed by the American Society of Science and Engineering.)
\end{abstract}

Key Words: Maximum Correlated Kurtosis Deconvolution (MCKD), Incipient Fault Enhancement, Fault Feature Extraction

\section{INTRODUCTION}

Fault detection and diagnosis of rotating machinery, with the purpose of early detection of potential faults, will be of great significance to guarantee efficient operation of machines [1-3]. In addition, they enhance the economic profits of enterprises and safeguard public security $[4,5]$.

Vibration signal analysis is currently a widely applied technique in mechanical fault diagnosis [6]. However, for incipient faults in rotating machinery, the fault information in the vibration signals is generally quite weak due to the effects of noise, complex transmission paths and signal attenuation, etc [7]. This makes the incipient fault particularly difficult to diagnose $[8,9]$. Consequently, whether the weak fault signals can be effectively enhanced is quite crucial to incipient fault diagnosis of rotating machinery, since it directly determines whether the fault features can be accurately extracted [10].

In many engineering applications, demodulated resonance technique is commonly used in the analysis of fault vibration signals [11]. Specifically, narrow-band filtering, where the resonant frequency should be adopted as the centre frequency, is performed to the vibration signals of rotating machinery [12]. Then the pulse train with identical frequency to as that of the impulsive fault signals is extracted by means of envelope detection. Nevertheless, when demodulated resonance method is used, one should set the centre frequency and the band of the filter in advance, the choice of which directly affects the diagnosis results. Using another common method, namely, the feature extraction of incipient faults of rotating machines based on wavelet envelope analysis, we have also obtained many favourable results [13, 14]. However, the selection of wavelet basis function lacks adaptability [15]. 
As an adaptive technique, EEMD has several advantages in processing the nonlinear dynamic signals and thus has been widely applied to the feature extraction of incipient faults $[16,17]$. Guo et al. [18] proposed a signal processing method based on EEMD and spectral kurtosis. This method was then applied to bearing fault diagnosis, and the results indicated that the fault could be located more quickly. Based on EEMD and wavelet neural network, Lei et al. [19] developed another fault diagnosis method for bearings, and the experimental results demonstrate that this method is applicable for hybrid fault identification. In order to achieve an adaptive fault diagnosis for rotating machines, Zhou et al [20] combined EEMD and adaptive demodulated resonance technique (ADRT). They then determined the central frequency and band of band-pass filter, and finally conducted energy operator demodulation on the filtered signals.

Firstly proposed by Wiggins, minimum entropy deconvolution (MED) [21] aims at extracting the mutational components of data. According to the minimum entropy criterion, the original simple pattern or the certainty of signals can be recovered by iterating and updating the filter. This technique minimizes the entropy and enhances the pulses in the signals. In 2007, Sawalhi et al. [22] indicated that MED could be used in gear fault diagnosis. By introducing the autoregressive (AR) model, Sawalhi advanced ARMED (AR method followed by MED) for gear fault diagnosis under the condition of noise interference. However, in practical applications, only a few large sharp pulses can be enhanced, leading to the loss of fault impacts. To solve the above described problem, McDonald et al. further proposed MCKD [23], where the correlated kurtosis is adopted as the evaluation index. With the periodicity of the impulsive components of signals fully taken into account, the deconvolution is achieved in iterative process, and the continuous pulses hidden in intense noise can then be magnified.

As described above, the incipient fault signals in rotating machinery are too weak to be detected under the interference of environmental noise. In this paper, considering the adaptivity of EEMD and the sensitivity of MCKD in the analysis of incipient impulsive faults, a novel fault diagnosis strategy for rotating machines is proposed. The following sections are organized as follows: in Section 2 and Section 3, the basic theories of EEMD, MED and MCKD are briefly reviewed; the proposed fault diagnosis strategy for rotating machines based on EEMD-MCKD is demonstrated in Section 4 and the feasibility of the proposed method is experimentally verified in Section 5; and finally, Section 6 presents the conclusions.

\section{EEMD ALGORITHM}

Aiming at solving the mode mixing problem in empirical mode decomposition (EMD) [24], Wu and Huang proposed EEMD in 2009 [25]. This method employs noise analysis in EMD, so that the mixing can be effectively suppressed. EEMD is very suitable for the detection of nonlinear, dynamic and weak signals. In principle, by using EEMD, the signals can become continuous at different characteristic time scales by applying superimposed white Gaussian noise. In this way the problem of mode mixing can be successfully addressed.

The detailed procedure of EEMD is presented as follows:

Step 1: The white Gaussian noise $n_{i}(t)$ with mean value of zero and amplitude equal to standard deviation is added to the original signal for $M$ times $(M>1)$ :

$$
x_{i}(t)=x(t)+n_{i}(t), i=1,2, \ldots, M
$$

Step 2: Perform EMD decomposition on $x_{i}(t)$, and $K \operatorname{IMFs} c_{i j}(t)(j=1,2, \ldots, K)$ and one residue $r_{i}(t)$ can be obtained. Specifically, $c_{i j}(t)$ refers to the $j^{\text {th }}$ IMF of the signal $x_{i}(t)$ in which the white Gaussian noise has been added for the $i^{\text {th }}$ time. 
Step 3: Since the statistical mean value of an uncorrelated random sequence is zero, the IMFs are averaged, so that the influence induced by the addition of white Gaussian noise for several times can be eliminated. Finally, the IMFs and the residue with EEMD can be expressed as:

$$
\begin{gathered}
c_{j}(t)=\frac{1}{M} \sum_{i=1}^{M} c_{i j}(t) \\
r(t)=\frac{1}{M} \sum_{i=1}^{M} r_{i}(t)
\end{gathered}
$$

where $c_{j}(t)$ denotes the $j$ th IMF component of the original signal with EEMD.

As stated above, $K$ IMFs and one residue $r(t)$ can be obtained. And the original signal can be reconstructed as follows:

$$
x(t)=\sum_{j=1}^{K} c_{j}(t)+r(t)
$$

\section{MED AND MCKD}

\subsection{MED}

To effectively extract useful information from seismic data, Wiggins proposed the MED, which is a systematic identification method. When performing MED, we seek the optimal filter coefficient $f$ by using an objective function in such a way that the kurtosis of the enhanced signal $y$ is the maximum. For example, $y=(x+e) * h$ (see Fig. 1), where $x$ denotes the input impact sequence, $e$ denotes the noise, and $y$ denotes the response after suffering from environmental impacts and attenuation, MED searches the optimal inverse filter $f$ so that the output $y$ is approximately equal to $x$.

\section{Optimal inverse filter $f$}

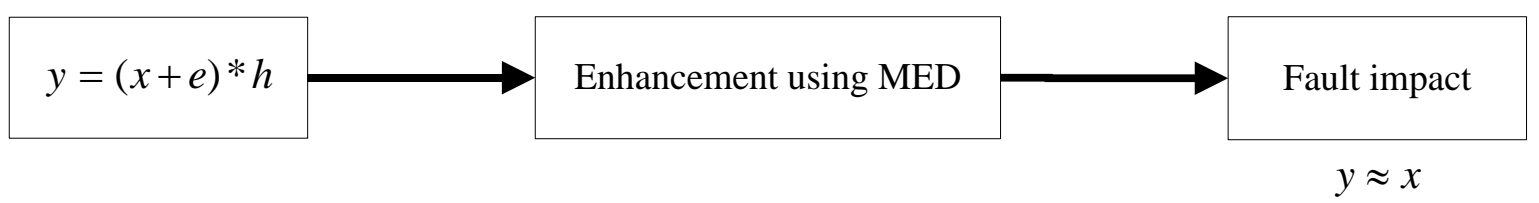

Figure 1: Deconvolution process in MED.

In MED, the objective function can be defined as:

$$
o_{4}(f[l])=\frac{\sum_{n=1}^{N} y^{4}[n]}{\left[\sum_{n=1}^{N} y^{2}[n]\right]^{2}}
$$

During the deconvolution process, the inverse filter can be expressed as:

$$
y(n)=\sum_{l=1}^{L} f[l] e_{1}[n-l]
$$

The optimal filter coefficient $f$ can be obtained when the objective function $o_{4}(f[l])$ achieves: 


$$
\frac{\partial\left(o_{4}(f[l])\right)}{\partial(f[l])}=0
$$

By substituting $\frac{\partial y[n]}{\partial(f[l])}=e_{1}[n-l]$ into eqs. (6) and (7), we can obtain the following expression:

$$
\underbrace{\left[\sum_{n=1}^{N} y^{2}[n] / \sum_{n=1}^{N} y^{4}[n]\right] \sum_{n=1}^{N} y^{3}[n] e_{1}[n-l]}_{\mathrm{b}}=\underbrace{\sum_{p=1}^{L} f[p]}_{f} \underbrace{\sum_{n=1}^{N} e_{1}[n-l] e_{1}[n-p]}_{A}
$$

Eq. (8) can also be expressed as:

$$
b=A f
$$

\subsection{MCKD}

In MED, the variation of maximum kurtosis is adopted as the terminating criterion of iteration, and it enhances the impulsive pulses. MED is therefore ideal for feature extraction of impulsive faults in rotating machines. Nevertheless, in practical applications, only a minority of sharp pulses can be enhanced, resulting in great losses of fault impacts signals. To solve this problem, McDonald made an improvement on MED. By selecting a finite impulse response (FIR) filter, the correlated kurtosis of the signal is maximized in line with the convergence criterion of iteration, which is referred to as the MCKD technique. In the following, the specific definitions will be described in detail.

Define the correlated kurtosis of first-shift as:

$$
C K_{1}(T)=\sum_{n=1}^{N}\left(y_{n} y_{n-T}\right)^{2} /\left(\sum_{n=1}^{N} y_{n}^{2}\right)^{2}
$$

where $y_{n}$ denotes a periodic signal with a period $T$.

Then, the correlated kurtosis of $M$-shift can be defined as:

$$
C K_{\mathrm{M}}(T)=\sum_{n=1}^{N}\left(\prod_{m=0}^{M} y_{n-m T}\right)^{2} /\left(\sum_{n=1}^{N} y_{n}^{2}\right)^{M+1}
$$

where $y_{n}=\sum_{k=1}^{L} \int f_{k} x_{n-k+1}, N$ denotes the sample number of the input signal $\vec{x}$, and $L$ denotes the length of a FIR filter. It should be noted that $n \neq 1,2, \ldots, N$ when $x_{n}=0$ and $y_{n}=0$.

The output signal $f_{k}$ from the filter bank is periodic, and the period approximately equals to $T$. The signal has high kurtosis value. When $T=0$ and $M=1, C K$ is the kurtosis criterion in MED.

By using the correlated kurtosis deconvolution of high-order shift, the capability of fault detection can be improved. The number of serial pulse in deconvolution can be enhanced by increasing the number of shift. Taking the maximum of Correlated kurtosis as the objective function, the filter coefficient $f_{k}$ can be calculated by taking the derivative with respect to the numerator and the denominator of $C K_{M}$ :

$$
M C K D_{\mathrm{M}}(T)=\max _{\vec{f}} C K_{M}(T)=\max _{\vec{f}} \sum_{n=1}^{N}\left(\prod_{m=0}^{M} y_{n-m T}\right)^{2} /\left(\sum_{n=1}^{N} y_{n}^{2}\right)^{M+1}
$$


where $\vec{f}$ denotes the filter bank and $M$ denotes the number of shifted period of the signals, the latter also called as shift number.

Then we take the derivative of the numerator and denominator in eq. (12), and set $\frac{d}{d f_{k}} C K_{M}(T)=0$. When $k=1,2, \ldots, L$, it is converted to the matrix, and the iterative solution can be expressed as:

$$
\vec{f}=\frac{\|\vec{y}\|}{2\|\vec{\beta}\|}\left(X_{0} X_{0}^{T}\right)^{-1} \sum_{m=0}^{M}\left(X_{m T} \vec{\alpha}_{m}\right)
$$

where $\vec{\alpha}_{m}=\left[\begin{array}{c}y_{1-m T}^{-1}\left(y_{1}^{2} y_{1-T}^{2} \cdots y_{1-M T}^{2}\right) \\ y_{2-m T}^{-1}\left(y_{2}^{2} y_{2-T}^{2} \cdots y_{2-M T}^{2}\right) \\ \vdots \\ y_{N-m T}^{-1}\left(y_{N}^{2} y_{N-T}^{2} \cdots y_{N-M T}^{2}\right)\end{array}\right] \quad \vec{\beta}=\left[\begin{array}{c}y_{1} y_{1-T} \cdots y_{1-M T} \\ y_{2} y_{2-T} \cdots y_{2-M T} \\ \vdots \\ y_{N} y_{N-T} \cdots y_{N-M T}\end{array}\right]$

$$
x_{r}=\left[\begin{array}{ccccc}
x_{1-r} & x_{2-r} & x_{3-r} & \cdots & x_{N-r} \\
0 & x_{1-r} & x_{2-r} & \cdots & x_{N-1-r} \\
0 & & x_{1-r} & \cdots & x_{N-2-r} \\
\vdots & \vdots & \vdots & & \vdots \\
0 & 0 & 0 & \cdots & x_{N-L-r+1}
\end{array}\right] \quad r=\left[\begin{array}{lllll}
0 & T & 2 T & \cdots & m T
\end{array}\right]
$$

According to the analysis above, the iteration of the MCKD-based fault enhancement consists of the following steps:

Step 1: Select the period $T$ and calculate $X_{T}, X_{0}^{T}$ and $\left(X_{0} X_{0}^{T}\right)^{-1}$ from input signal $\vec{x}$;

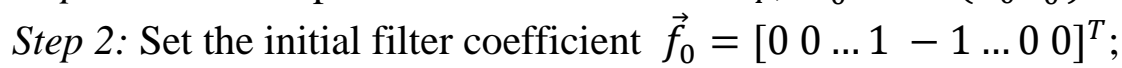

Step 3: Calculate the filtered output $\vec{y}$;

Step 4: Calculate $\vec{\alpha}_{m}$ and $\vec{\beta}$ from $\vec{y}$;

Step 5: Calculate the new filter coefficient $\vec{f}_{1}$ from eq. (13);

Step 6: Calculate the iterative error according to the following equation:

$$
\text { err }=\left(f^{(1)}-\mu f^{(0)}\right) / \mu f^{(0)}
$$

If $E(e r r)$ is smaller than the given iterative error, the calculation will be terminated; otherwise return to Step 3 and continue the calculation.

\section{A NOVEL FAULT DIAGNOSIS STRAGETY BASED ON EEMD AND MCKD}

For the original signal containing strong background noise, several IMFs with different vibration modes can be obtained with EEMD. The original signal is decomposed according to its frequency. In addition to a great amount of fault information, the modulated signals of high-frequency IMFs also contain much noise information. Therefore, the impulsive signals which reflect the mechanical faults can hardly be effectively extracted. In this paper, a novel feature extraction method for weak fault signals based on MCKD enhancement and EEMD is proposed, with the specific flowchart provided in Fig. 2. Firstly, EEMD is performed on the collected sample fault vibration signals of rotating machines, and various IMFs reflecting the fault conditions can be obtained. Based on correlation coefficient and kurtosis criterion, the IMFs which contain the fault information of rotating machines are selected for further reconstruction of original signal. MCKD-based adaptive enhancement is then performed on 
the reconstructed signal. Finally, the characteristic frequency of fault can be extracted from envelope spectrum, and the diagnosis results are output.

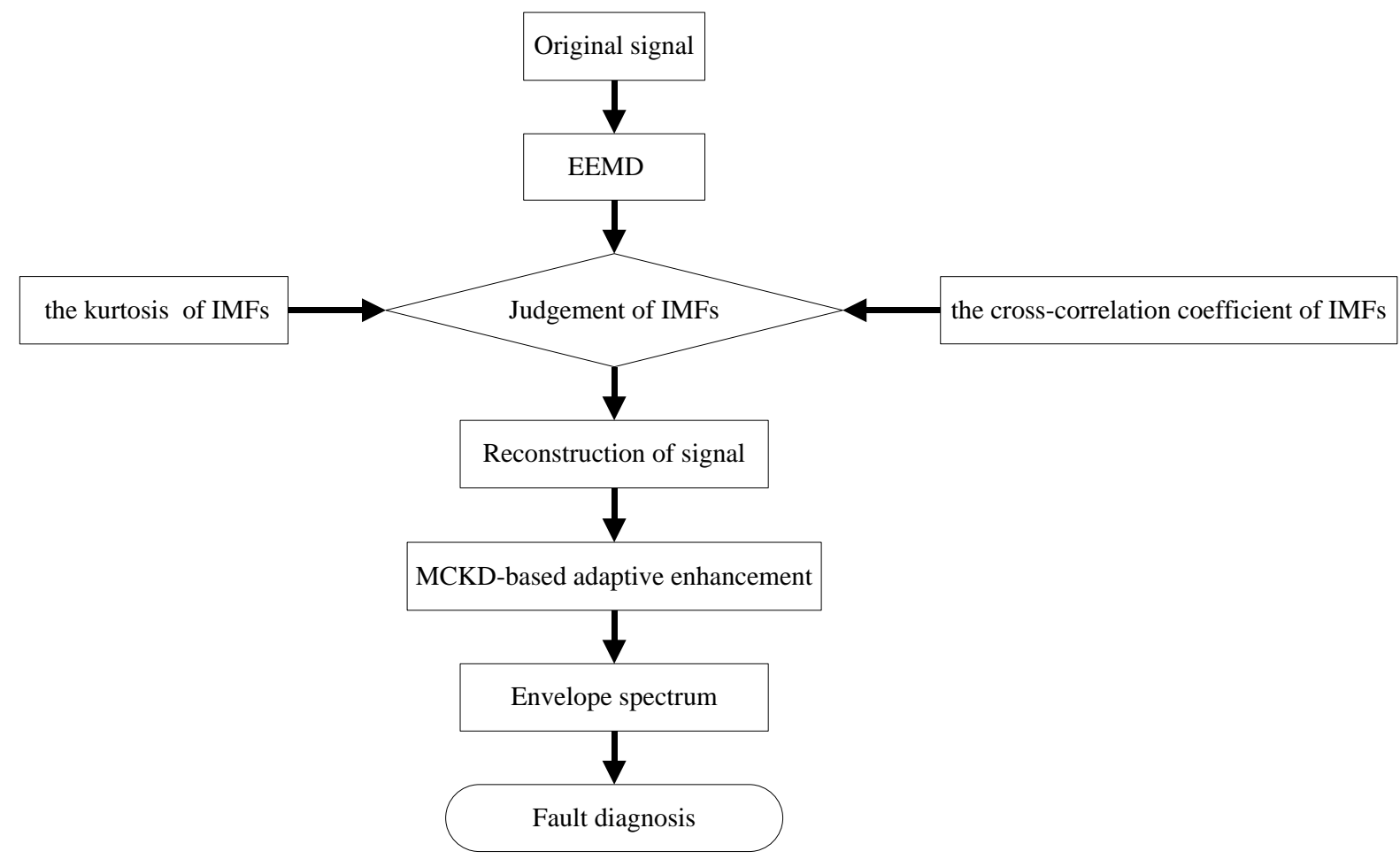

Figure 2: The flow chart of the proposed novel fault diagnosis strategy based on EEMD and MCKD.

\section{EXPERIMENTS}

As shown in Fig. 3, the experimental measurement system is composed of a speed-adjustablemotor, a coupling, a single reduction gearbox, a magnetic powder brake, a vibration acceleration sensor and a signal analyser. The tooth numbers of input and output gears are 55 and 75 , and the modulus is 2 .

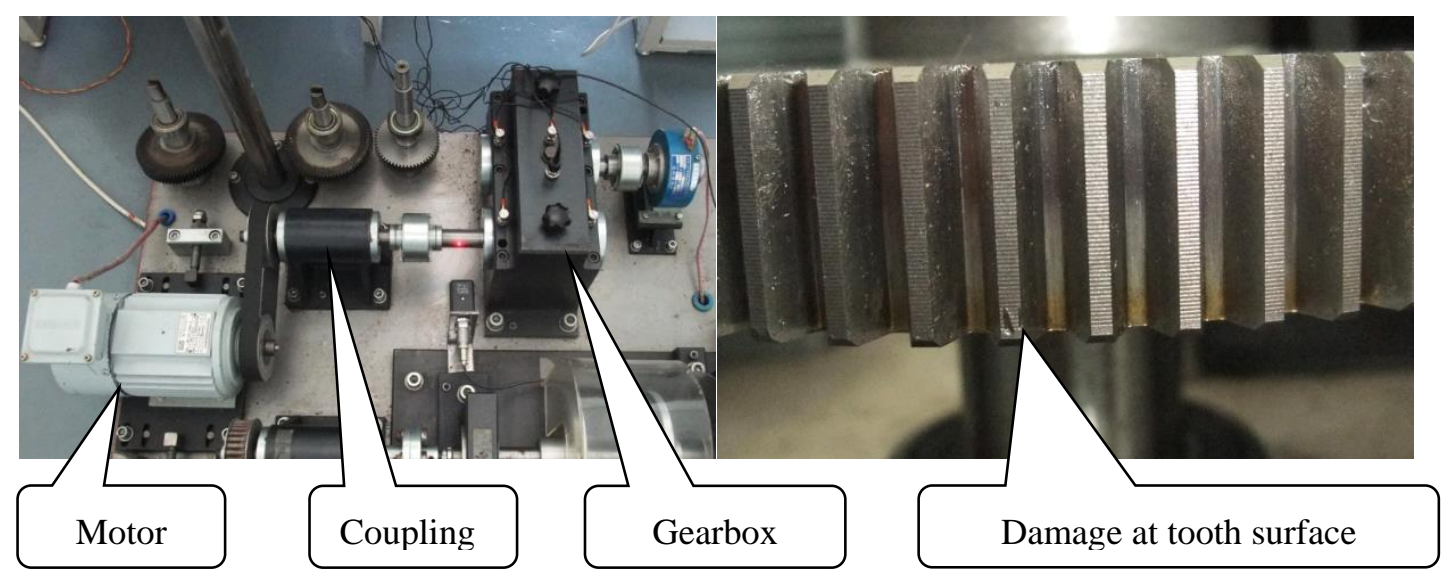

Figure 3: The experimental device and the fault gear.

A small damage is processed at the tooth surface of output gear (in Fig. 3). The rotation speed of the motor is $800 \mathrm{r} / \mathrm{min}$ and the load of the magnetic powder brake is $100 \mathrm{~N}$. The vibration signals of the gearbox are collected using the acceleration sensor (KistlerICP, Kistler Instrument AG, Switzerland) and then acquired using the data acquisition equipment 
(DP/INV306U), with the sampling frequency of $10 \mathrm{kHz}$. Fig. 4 shows the original fault signal of the damage at tooth surface of output gear.

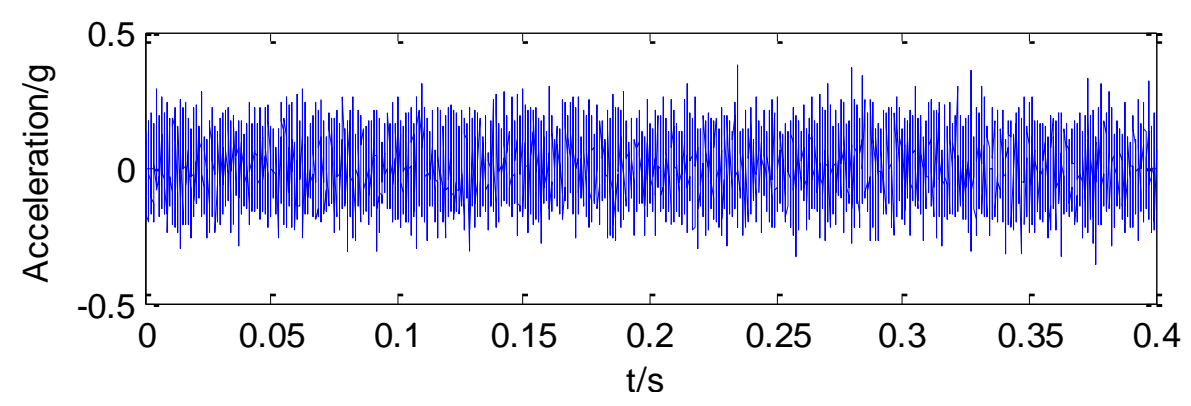

Figure 4: Original fault signal of the damage at tooth surface.

First, the EEMD method is used to analyse the original signal, and the first five IMFs and the residue are given in Fig. 5. Then we calculate the correlation coefficients between the IMFs and the original signal to evaluate the correlation between them. The IMFs with high degrees of correlation are then selected to extract fault features.

As shown in Table I, the first three IMFs are retained to reconstruct the original signal based on the correlation coefficients. Fig. 6 presents the reconstructed signal of outer ring. We also calculated the kurtosis values of the fault signal with the MCKD-based adaptive enhancement, as listed in Table II. It can be found that, the kurtosis value increases from 2.136 to 5.273 after the enhancement. Figs. 6 and 7 refer to the reconstructed fault signals without and with the MCKD-based enhancement, respectively. By comparison, we can find that the impulsive components in the fault signal have been enhanced with the MCKD method.

For the original signal, the IMFs are selected based on the calculated correlation coefficient and kurtosis, and then used for reconstructing the fault signal. Figs. 8 and 9 show the envelope spectrums of the reconstructed signal with EEMD and EEMD-MED, respectively. It can be noted that by using this reconstruction method, the characteristic frequency of the fault is not obvious. For the purpose of comparison, Fig. 10 shows the envelope spectrum of the fault signal reconstructed using the proposed method and the amplitude of the spectrum line at the fault characteristic frequency has been significantly enhanced.

To further verify the effectiveness and stability of the proposed method, 120 samples corresponding to each operating states of the gearbox are collected, and each sample has 10000 points. 30 samples are randomly selected as the training sample set, and the other 90 samples are adopted as the testing sample set. The fault samples are then input to a multi-kernel support vector machine classifier for identification, and the results are listed in Table III. It can be found that the accuracy of the EEMD-MCKD method is apparently higher than the results based on either the EEMD or the EEMD-MED method.

Table I: The results of correlation coefficient and kurtosis among the IMFs.

\begin{tabular}{|l|c|c|c|c|c|}
\hline IMF component & 1 & 2 & 3 & 4 & 5 \\
\hline Correlation coefficient & 0.584 & 0.628 & 0.431 & 0.189 & 0.172 \\
\hline Kurtosis & 2.633 & 3.358 & 2.645 & 1.528 & 1.812 \\
\hline
\end{tabular}

Table II: The kurtosis values of the original signal and the enhanced signal.

\begin{tabular}{|c|c|c|}
\cline { 2 - 3 } \multicolumn{1}{c|}{} & Original signal & Enhanced signal using MED \\
\hline Kurtosis & 2.136 & 5.273 \\
\hline
\end{tabular}



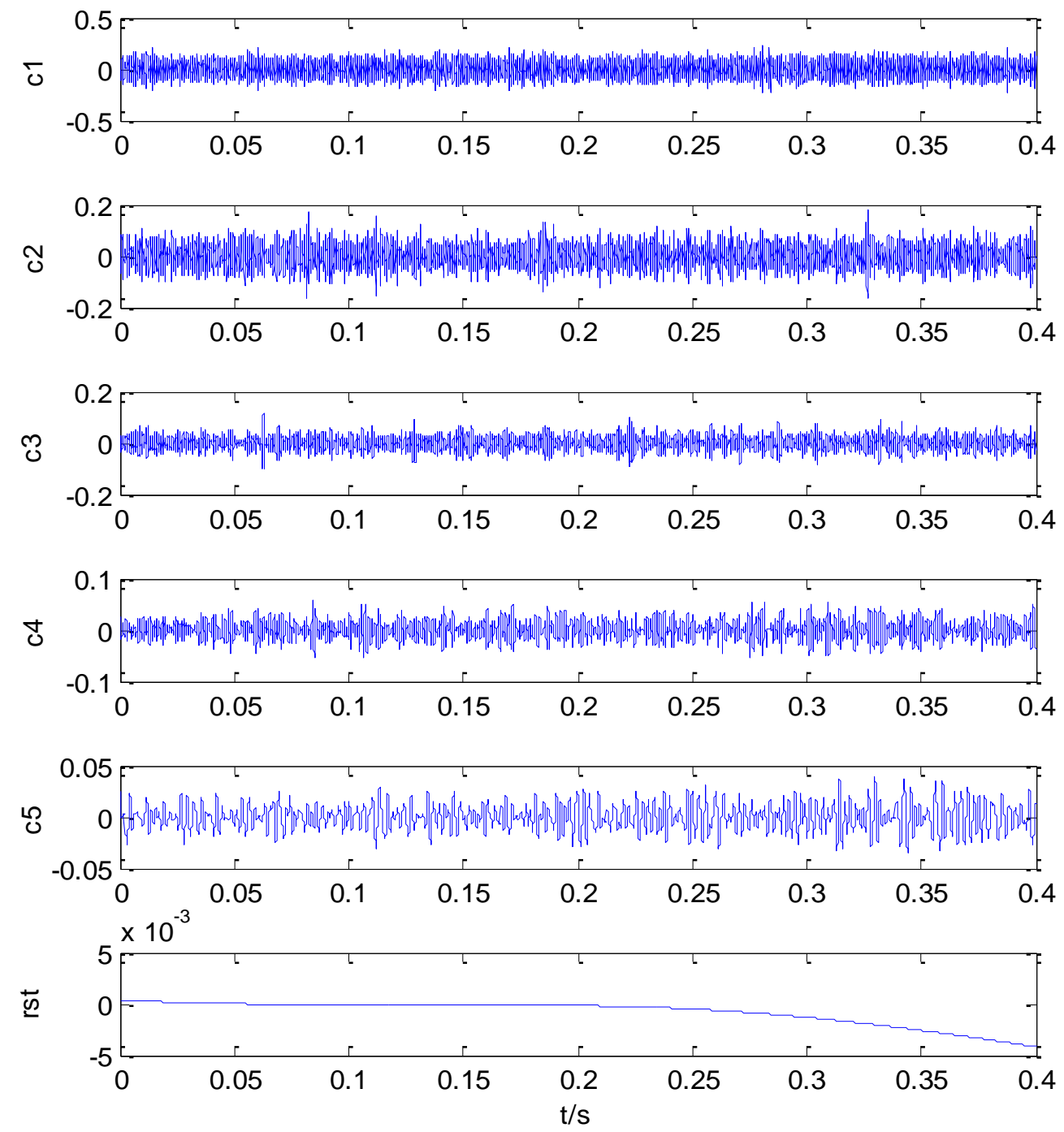

Figure 5: The first five IMFs and the residue decomposed by EEMD.

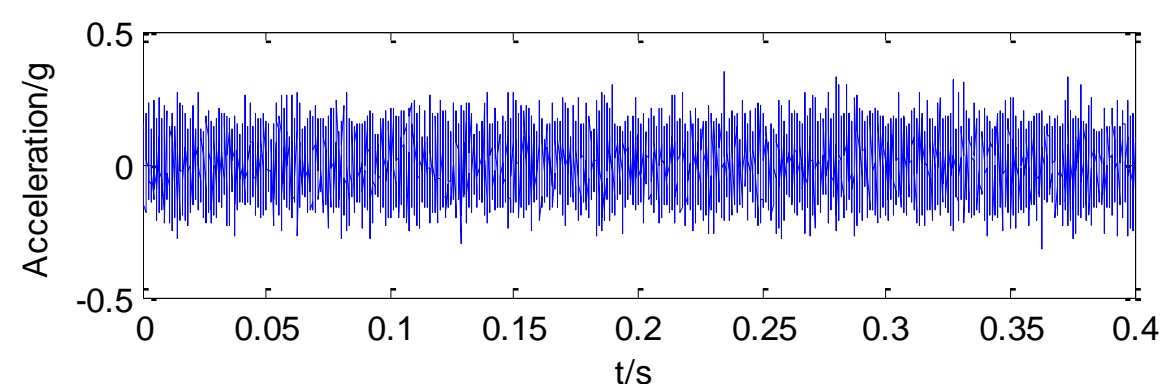

Figure 6: Original reconstructed fault signal.

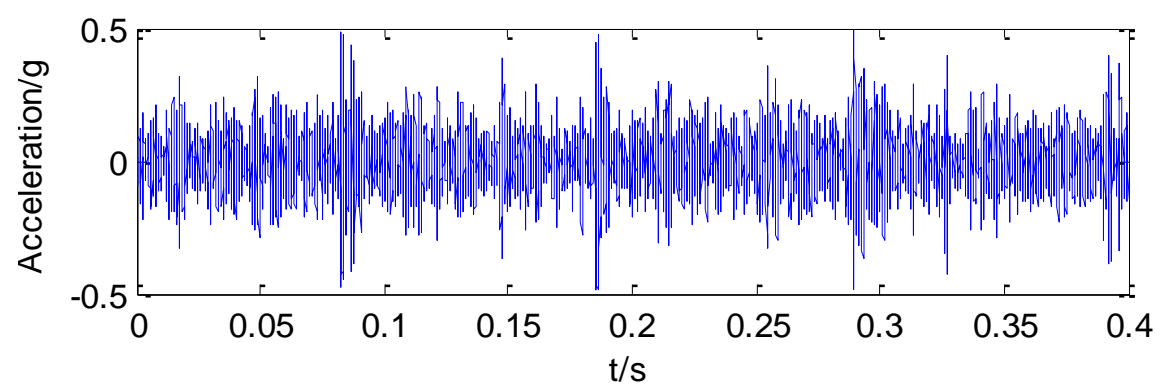

Figure 7: Reconstructed fault signal from the IMFs with MCKD enhancement. 


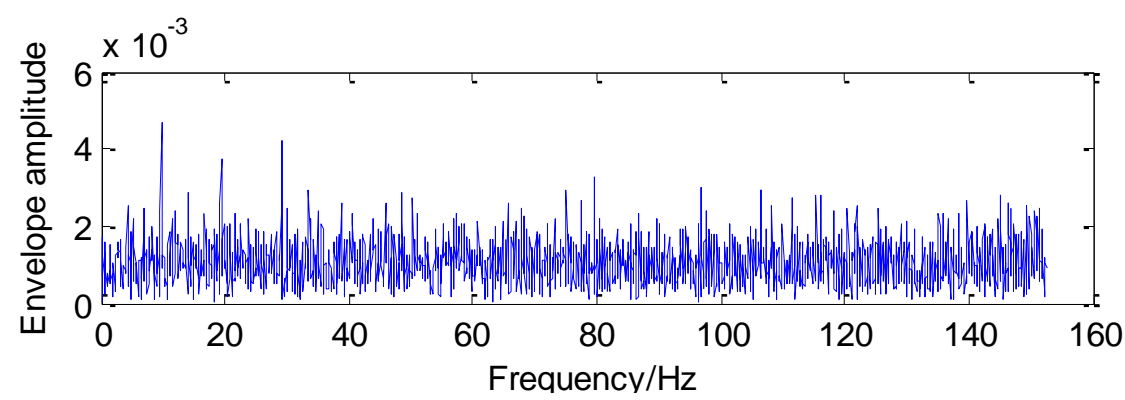

Figure 8: Envelope spectrum of the original reconstructed signal with EEMD.

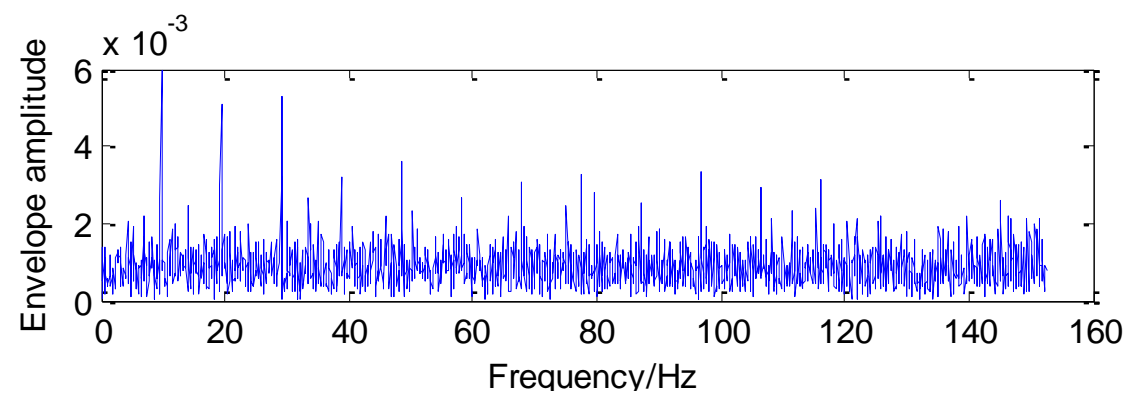

Figure 9: Envelope spectrum of the original reconstructed signal with EEMD-MED.

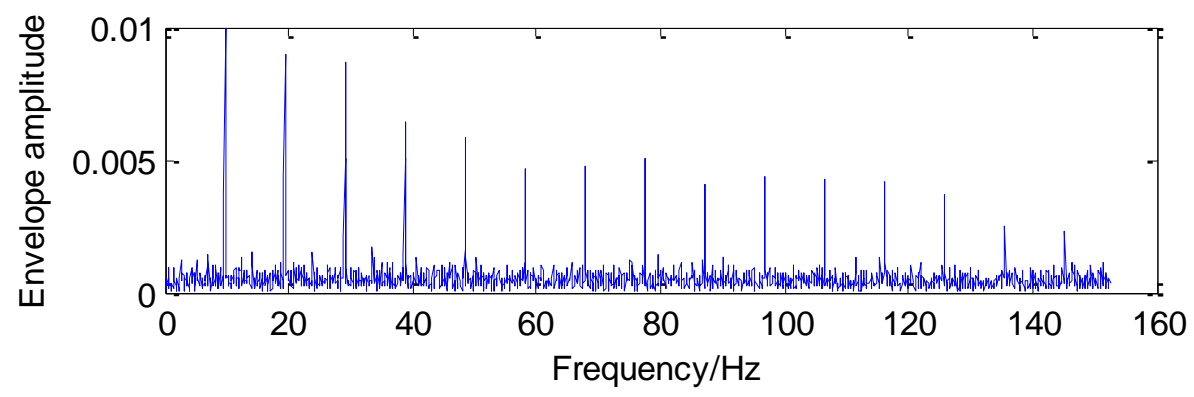

Figure 10: Envelope spectrum of the reconstructed signal with the proposed method.

Table III: Comparison of the classification accuracy of different models.

\begin{tabular}{|l|c|c|c|}
\hline Diagnostic model & EEMD-MCKD & EEMD-MED & EEMD \\
\hline $\begin{array}{l}\text { Average identification } \\
\text { accuracy }(\%)\end{array}$ & $87.8 \%$ & $78.9 \%$ & $71.1 \%$ \\
\hline
\end{tabular}

To validate the robustness and generalizability of the diagnostic model, the original faulted vibration signal $x_{k}$ is contaminated by adding random noises. The signal with noise can be expressed as $x_{k}^{\prime}=[1+\alpha \cdot \operatorname{rand}(1)] x_{k}$, and $\alpha$ is set to be $0.1,0.2$ and 0.3 in this work respectively. The characteristic values of noisy signal are input to three diagnostic models, namely, EEMD-MCKD, EEMD-MED and EEMD, with the recognized results shown in Fig. 11. It can be noticed from Fig. 11 that, the diagnostic model based on the EEMD-MCKD not only provides higher accuracy, but also has better anti-noise performances. Even when the extracted fault characteristics are interfered by a certain degree of noise, the fault identification results are still desirable using this diagnostic model. In practical engineering applications, due to the effects of the complex environmental factors, the identification results of faults using the models based on EEMD and EEMD-MED are not as ideal as expected in most cases. As conclusion, the model based on EEMD-MCKD has a certain anti-interference 
ability to noise and a strong generation capability, and thus can be successfully applied in engineering applications.

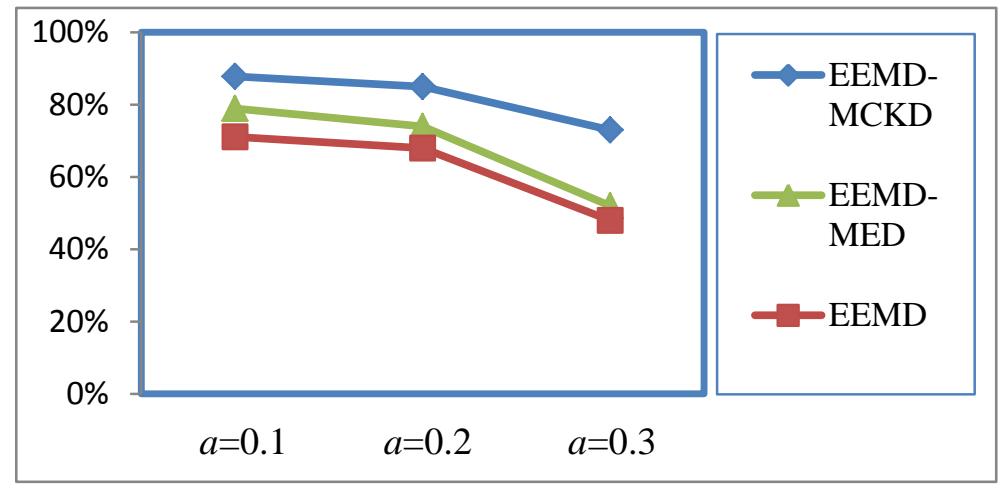

Figure 11: The anti-noise ability comparison of different diagnosis models.

\section{CONCLUSIONS}

In this paper, a novel fault diagnosis method for rotating machinery based on EEMD and MCKD is proposed. When the signal is affected by the noise or the fault feature is not apparent, the weak fault feature can hardly be extracted from the IMFs. By using MCKD, the weak impulsive characteristics can be effectively enhanced. MCKD technique exhibits remarkable advantages in suppressing the noise and extracting the periodic impulsive pulses. From the IMFs, the original signal is reconstructed based on the calculated correlation coefficient and kurtosis. Subsequently, the impulsive pulses in reconstructed signal are enhanced through the usage of MCKD-based adaptive technique, and the weak fault features can be effectively extracted from the envelope spectrum. The results indicate that, the fault impulsive information in the reconstructed signal can be significantly enhanced by using the proposed method, and thus the weak features in incipient faults can be effectively extracted. Moreover, the effectiveness of the proposed method is validated by the detection of gear fault at the tooth surface.

\section{ACKNOWLEDGEMENTS}

This research is supported by the National Natural Science Foundation of China (Project No. 51375514), Chongqing Commission of Science and Technology Research Projects (Project No. KJ1401303) and the Research Foundation of Chongqing University of Science \& Technology (Project No. CK2015Z19). Finally, the authors are very grateful to the reviewers for their helpful comments and constructive suggestions.

\section{REFERENCES}

[1] Kappaganthu, K.; Nataraj, C. (2011). Feature selection for fault detection in rolling element bearings using mutual information, Journal of Vibration and Acoustics, Vol. 133, No. 6, Paper No. 061001, 11 pages, doi: $10.1115 / 1.4003400$

[2] Bin, G. F.; Gao, J. J.; Li, X. J.; Dhillon, B. S. (2012). Early fault diagnosis of rotating machinery based on wavelet packets - Empirical mode decomposition feature extraction and neural network, Mechanical Systems and Signal Processing, Vol. 27, 696-711, doi:10.1016/j.ymssp.2011.08.002

[3] Ming, Y.; Chen, J.; Dong, G. (2011). Weak fault feature extraction of rolling bearing based on cyclic Wiener filter and envelope spectrum, Mechanical Systems and Signal Processing, Vol. 25, No. 5, 1773-1785, doi:10.1016/j.ymssp.2010.12.002 
[4] Gelman, L.; Murray, B.; Patel, T. H.; Thomson, A. (2015). Novel wavelet technology for vibration condition monitoring of rolling element bearings, Insight - Non-Destructive Testing and Condition Monitoring, Vol. 57, No. 1, 40-47, doi:10.1784/insi.2015.57.1.40

[5] Hameed, Z.; Ahn, S. H.; Cho, Y. M. (2010). Practical aspects of a condition monitoring system for a wind turbine with emphasis on its design, system architecture, testing and installation, Renewable Energy, Vol. 35, No. 5, 879-894, doi:10.1016/j.renene.2009.10.031

[6] Chen, H. X.; Chua, P. S. K.; Lim, G. H. (2008). Fault degradation assessment of water hydraulic motor by impulse vibration signal with Wavelet Packet Analysis and Kolmogorov-Smirnov Test, Mechanical Systems and Signal Processing, Vol. 22, No. 7, 1670-1684, doi:10.1016/j.ymssp. 2008.01.009

[7] Zarei, J.; Tajeddini, M. A.; Karimi, H. R. (2014). Vibration analysis for bearing fault detection and classification using an intelligent filter, Mechatronics, Vol. 24, No. 2, 151-157, doi:10.1016/j.mechatronics.2014.01.003

[8] Muruganatham, B.; Sanjith, M. A.; Krishnakumar, B.; Satya Murty, S. A. V. (2013). Roller element bearing fault diagnosis using singular spectrum analysis, Mechanical Systems and Signal Processing, Vol. 35, No. 1-2, 150-166, doi:10.1016/j.ymssp.2012.08.019

[9] Van, M.; Kang, H.-J.; Shin, K.-S. (2014). Rolling element bearing fault diagnosis based on non-local means de-noising and empirical mode decomposition, IET Science, Measurement and Technology, Vol. 8, No. 6, 571-578, doi:10.1049/iet-smt.2014.0023

[10] Garcia-Perez, A.; de Jesus Romero-Troncoso, R.; Cabal-Yepez, E.; Osornio-Rios, R. A. (2011). The application of high-resolution spectral analysis for identifying multiple combined faults in induction motors, IEEE Transactions on Industrial Electronics, Vol. 58, No. 5, 2002-2010, doi:10.1109/TIE.2010.2051398

[11] Hou, S.; Li, Y.; Wang, Z. (2010). A resonance demodulation method based on harmonic wavelet transform for rolling bearing fault diagnosis, Structural Health Monitoring, Vol. 9, No. 4, 297-308, doi:10.1177/1475921709352144

[12] Zhang, Y.; Randall, R. B. (2009). Rolling element bearing fault diagnosis based on the combination of genetic algorithms and fast kurtogram, Mechanical Systems and Signal Processing, Vol. 23, No. 5, 1509-1517, doi:10.1016/j.ymssp.2009.02.003

[13] Yan, R.; Gao, R. X. (2009). Multi-scale enveloping spectrogram for vibration analysis in bearing defect diagnosis, Tribology International, Vol. 42, No. 2, 293-302, doi:10.1016/j.triboint. 2008.06.013

[14] Jena, D. P.; Panigrahi, S. N.; Kumar, R. (2013). Gear fault identification and localization using analytic wavelet transform of vibration signal, Measurement, Vol. 46, No. 3, 1115-1124, doi:10.1016/j.measurement.2012.11.010

[15] Zhang, S.; Zhang, Y.; Zhu, J. (2015). Rolling element-bearing feature extraction based on combined wavelets and quantum-behaved particle swarm optimization, Journal of Mechanical Science and Technology, Vol. 29, No. 2, 605-610, doi:10.1007/s12206-015-0120-3

[16] Jiang, H.; Li, C.; Li, H. (2013). An improved EEMD with multiwavelet packet for rotating machinery multi-fault diagnosis, Mechanical Systems and Signal Processing, Vol. 36, No. 2, 225-239, doi:10.1016/j.ymssp.2012.12.010

[17] Lei, Y.; He, Z.; Zi, Y. (2009). Application of the EEMD method to rotor fault diagnosis of rotating machinery, Mechanical Systems and Signal Processing, Vol. 23, No. 4, 1327-1338, doi:10.1016/j.ymssp.2008.11.005

[18] Guo, W.; Tse, P. W.; Djordjevich, A. (2012). Faulty bearing signal recovery from large noise using a hybrid method based on spectral kurtosis and ensemble empirical mode decomposition, Measurement, Vol. 45, No. 5, 1308-1322, doi:10.1016/j.measurement.2012.01.001

[19] Lei, Y.; He, Z.; Zi, Y. (2011). EEMD method and WNN for fault diagnosis of locomotive roller bearings, Expert Systems with Applications, Vol. 38, No. 6, 7334-7341, doi:10.1016/j.eswa. 2010.12.095

[20] Zhou, Z.; Zhu, Y. S.; Zhang, Y. Y.; Zhu, C. F.; Wang, P. (2013). Adaptive fault diagnosis of rolling bearings based on EEMD and demodulated resonance, Journal of Vibration and Shock, Vol. 32, No. 2, 76-80

[21] Wiggins, R. A. (1978). Minimum entropy deconvolution, Geoexploration, Vol. 16, No. 1-2, 21-35, doi: $10.1016 / 0016-7142(78) 90005-4$ 
[22] Sawalhi, N.; Randall, R. B.; Endo, H. (2007). The enhancement of fault detection and diagnosis in rolling element bearings using minimum entropy deconvolution combined with spectral kurtosis, Mechanical Systems and Signal Processing, Vol. 21, No. 6, 2616-2633, doi:10.1016/j.ymssp.2006.12.002

[23] McDonald, G. L.; Zhao, Q.; Zuo, M. J. (2012). Maximum correlated Kurtosis deconvolution and application on gear tooth chip fault detection, Mechanical Systems and Signal Processing, Vol. 33, 237-255, doi:10.1016/j.ymssp.2012.06.010

[24] Huang, N. E.; Shen, Z.; Long, S. R.; Wu, M. C.; Shih, H. H.; Zheng, Q.; Yen, N.-C.; Tung, C. C.; Liu, H. H. (1998). The empirical mode decomposition and the Hilbert spectrum for nonlinear and non-stationary time series analysis, Proceedings of the Royal Society A: Mathematical, Physical \& Engineering Sciences, Vol. 454, No. 1971, 903-995, doi:10.1098/rspa.1998.0193

[25] Wu, Z.; Huang, N. E. (2009). Ensemble empirical mode decomposition: a noise-assisted data analysis method, Advances in Adaptive Data Analysis, Vol. 1, No. 1, 1-41, doi:10.1142/ $\underline{\mathrm{S} 1793536909000047}$ 